Pacific

Journal of

Mathematics

WEAK PALEY-WIENER PROPERTY FOR COMPLETELY SOLVABLE LIE GROUPS

Gayatri Garimella 


\title{
WEAK PALEY-WIENER PROPERTY FOR COMPLETELY SOLVABLE LIE GROUPS
}

\author{
Gayatri GARIMELla
}

\begin{abstract}
We prove a weak Paley-Wiener property for completely solvable Lie groups, i.e. if the group Fourier transform of a measurable, bounded and compactly supported function vanishes on a set of positive Plancherel measure then the function itself vanishes almost everywhere on the group.
\end{abstract}

\section{Introduction.}

Let $G$ be a connected, simply connected, and completely solvable Lie group, with the Lie algebra $\mathfrak{g}$. Let $\mathfrak{g}^{*}$ be the dual of $\mathfrak{g}$. The equivalence classes of irreducible unitary representations $\widehat{G}$ of $G$ is parametrized by the coadjoint orbits $\mathfrak{g}^{*} / G$ via the Kirillov-Bernat bijective map $K: \widehat{G} \rightarrow \mathfrak{g}^{*} / G$. If $\rho \in \widehat{G}$ and $\ell \in K(\rho)$, then there exists an analytic subgroup $H$ of $G$ and a unitary character $\chi$ of $H$, such that $\left.\ell\right|_{\mathfrak{h}}=I d_{\chi}$, where $\mathfrak{h}$ is the Lie algebra of $H$. The induced representation $\rho=\operatorname{Ind}_{H}^{G} \chi$ is irreducible. Moreover, $K$ is a bijection. The image on $\mathfrak{g}^{*} / G$ of a measure equivalent to Lebesgue measure on $\mathfrak{g}^{*}$ gives a Plancherel measure on $\widehat{G}$.

Let $\phi$ be a bounded, measurable and compactly supported function on $\mathbb{R}^{n}$. By the classical Paley-Wiener theorem, the Fourier transform $\widehat{\phi}$ of $\phi$ extends to an entire function on $\mathbb{C}^{n}$. Using this we can conclude that if $\widehat{\phi}$ vanishes on a set of positive Plancherel measure which is nothing but the Lebesgue measure, then $\widehat{\phi}$ vanishes on the whole of $\mathbb{R}^{n}$. This in turn implies that $\phi=0$ on $\mathbb{R}^{n}$.

In the same spirit, for a completely solvable Lie group we will think of the following as a weak Paley-Wiener property:

Theorem. Let $G$ be a connected, simply connected, and completely solvable Lie group, with the unitary dual $\widehat{G}$. Let $\phi$ be a measurable, bounded, and compactly supported function (i.e $\phi \in L_{c}^{\infty}(G)$ ). Assume that there exists a subset $E \subset \widehat{G}$ with positive Plancherel measure such that $\widehat{\phi}_{\rho}=0$ for all $\rho \in E$ where $\widehat{\phi}_{\rho}$ is the group Fourier transform of $\phi$. Then $\phi=0$ almost everywhere on $G$.

In [GG1] we proved, the same theorem for nilpotent Lie groups, by induction on the dimension of $G$. To prove the above theorem, also by using 
induction on the dimension of $G$, we need a description of the dual space $\widehat{G}$ of $G$ and an explicit Plancherel measure on $\widehat{G}$. Here, we use the results of B.N. Currey $[\mathbf{C}]$, which are generalizations of the results of L. Pukanszky $[\mathbf{P u}]$ on nilpotent Lie groups concerning the Plancherel measure and the Plancherel formula.

\section{Preliminaries.}

Let $G$ be a connected, simply connected, and completely solvable Lie group, with the Lie algebra $\mathfrak{g}$. Let $\mathfrak{g}^{*}$ be the dual of $\mathfrak{g}$. We fix a basis $\mathfrak{B}=$ $\left\{X_{1}, \ldots, X_{n}\right\}$ of $\mathfrak{g}$, such that $\mathfrak{g}_{j}$ is spanned by the vectors $\left\{X_{1}, X_{2} \cdots, X_{j}\right\}$, $1 \leq j \leq n$ and $\mathfrak{g}_{0}=(0)$. As $G$ is completely solvable, there exists a chain of ideals

$$
0=\mathfrak{g}_{0} \subset \mathfrak{g}_{1} \subset \cdots \subset \mathfrak{g}_{i} \cdots \subset \mathfrak{g}_{n-1} \subset \mathfrak{g}_{n}=\mathfrak{g}
$$

of $\mathfrak{g}$, such that the dimension of $\mathfrak{g}_{i}$ be $i$ for all $1 \leq i \leq n$. Let $\mathfrak{B}^{*}=$ $\left\{X_{1}^{*}, \ldots, X_{n}^{*}\right\}$ be the dual basis of $\mathfrak{g}^{*}$. We fix a Lebesgue measure $d X$ on $\mathfrak{g}$, and a right Haar measure $d g$ on $G$ such that $d(\exp X)=j_{G}(X) d X$, where

$$
j_{G}(X)=\left|\operatorname{det}\left(\frac{1-e^{-a d X}}{a d X}\right)\right| .
$$

Let $\Delta$ be the modular function such that for all $g^{\prime} \in G, d\left(g g^{\prime}\right)=\Delta\left(g^{\prime}\right) d g$. Let $O$ be a coadjoint orbit in $\mathfrak{g}^{*}$ and $\ell \in O$. The bilinear form $B_{\ell}:(X, Y) \rightarrow$ $\ell([X, Y])$ defines a skew-symmetric and nondegenerate bilinear form on $\mathfrak{g} / \mathfrak{g}^{\ell}$. As the map $X \rightarrow X$. $\ell$ induces an isomorphism between $\mathfrak{g} / \mathfrak{g}^{\ell}$ and the tangent space of $O$ at $\ell$, the bilinear form $B_{\ell}$ defines a nondegenerate 2 -form $\omega_{\ell}$ on this tangent space. If $2 k$ is the dimension of $O$ we note that

$$
\beta_{O}=(2 \pi)^{-k}(k !)^{-1} \omega \wedge \cdots \wedge \omega \quad(k \text { times })
$$

the canonical measure on $O$. Lemma 3.2.2. in [DR], says us that there exists a nonzero rational function $\psi$ on $\mathfrak{g}^{*}$ such that $\psi(g . \ell)=\Delta(g)^{-1} \psi(\ell)$, $g \in G$, and $\ell \in \mathfrak{g}^{*}$. We fix one such $\psi$. There exists a unique measure $m_{\psi}$ on $\mathfrak{g}^{*} / G$ such that

$$
\int_{\mathfrak{g}^{*}} \phi(\ell)|\psi(\ell)| d \ell=\int_{\mathfrak{g}^{*} / G}\left(\int_{O} \phi(\ell) d \beta_{O}(\ell)\right) d m_{\psi}(O)
$$

for all Borel functions $\phi$ on $\mathfrak{g}^{*}$.

B.N. Currey $[\mathbf{C}]$ gave an explicit description of the measure $m_{\psi}$ with the help of the coadjoint orbits $\mathfrak{g}^{*} / G$. We recall the theorem proved by B.N. Currey in $[\mathbf{C}]$ which is the essential tool to prove our Paley-Wiener property:

Theorem 2.1. Let $G$ be a connected, simply connected, and completely solvable Lie group. There exists a Zariski open subset $U$ of $\mathfrak{g}^{*}$, a subset $J=\left\{j_{1}<j_{2}<\cdots<j_{2 k}\right\}$ of $\{1,2, \cdots, n\}$, a subset $M=\left\{j_{r_{1}}<j_{r_{2}}<\right.$ $\left.\cdots<j_{r_{a}}\right\}$ of $J$, for each $j$ in $M$ a real valued rational function $q_{j}$ (non 
vanishing on $U)$, and real analytic $P_{j}, 1 \leq j \leq n$ functions in the variables $w_{1}, w_{2}, \ldots, w_{2 k}, \ell_{1}, \ell_{2}, \cdots, \ell_{n}$ such that the following hold.

1) If a denotes the number of elements in $M$, for each $\epsilon \in\{1,-1\}^{a}$, the set

$$
U_{\epsilon}=\left\{\ell \in U \mid \text { sign of } q_{j_{r_{m}}}(\ell)=\epsilon_{m}, 1 \leq m \leq a\right\}
$$

is a non empty open subset in $\mathfrak{g}^{*}$.

2) Define $V \subset \mathbb{R}^{2 k}$ by $V=\prod R_{r}$, where $\left.R_{r}=\right] 0, \infty\left[\right.$ if $j_{r} \in M$ and $R_{r}=\mathbb{R}$ otherwise. Let $\epsilon \in\{1,-1\}^{a}$ and for $v \in V$, define $\epsilon v \in \mathbb{R}^{2 k}$ by $(\epsilon v)_{j}=\epsilon_{m} v_{j}$ if $j=j_{r_{m}} \in M$ and $(\epsilon v)_{j}=v_{j}$ otherwise. Then for each $\ell \in U_{\epsilon}$, the mapping $v \rightarrow \sum_{j} P_{j}(\epsilon v, \ell) X_{j}^{*}$ is a diffeomorphism of $V$ with the coadjoint orbit of $\ell$.

3) Define $W_{D}$ as the subspace spanned by the vectors $\left\{X_{i}^{*} \mid i \notin J\right\}$ and $W_{M}$ the subspace spanned by $\left\{X_{j}^{*} \mid j \in M\right\}$. Then the set

$$
W=\left\{\ell \in\left(W_{D} \oplus W_{M}\right) \cap U|| q_{j}(\ell) \mid=1, j \in M\right\}
$$

is a cross-section for the coadjoint orbits $U$. For each $j \in M$ the rational function $q_{j}$ is of the form $q_{j}(\ell)=\ell_{j}+p_{j}\left(\ell_{1}, \ell_{2}, \cdots, \ell_{j-1}\right)$, where $p_{j}$ is a rational function.

4) For each $\ell \in U$, let $\epsilon(\ell) \in\{1,-1\}^{a}$ such that $\ell \in U_{\epsilon(\ell)}$. Then the mapping $P: V \times W \rightarrow U$, defined by $P(v, \ell)=\sum_{j} P_{j}(\epsilon(\ell) v, \ell) X_{j}^{*}$, is a diffeomorphism.

B.N. Currey $[\mathbf{C}]$ proved that $m_{\psi}$ is a Plancherel measure on $W$.

The idea is to compute the measure $\psi(\ell) d l$ in termes of product measures on $V \times W$ and then, using Lemma 1.3 of $[\mathbf{C}]$, we can read off $m_{\psi}$ as a measure on $W$. We have to determine coordinates for $W$.

If the subset $M$ of $J$ is empty, then $W=W_{D} \cap U$ and the coordinates for $W$ are obtained by identifying $W_{D}$ with $\mathbb{R}^{n-2 k}$, which is the parametrization of $\mathfrak{g}^{*}$ in the nilpotent case. On the other hand, suppose that $M$ is non empty, and $a$ denotes the number of elements in $M$. From $[\mathbf{C}]$, for each $\epsilon \in\{1,-1\}^{a}$, there exists a non empty Zariski open subset $U_{\epsilon}$ of $U$ and $U$ is the disjoint union of the sets $U_{\epsilon}$. Let $\epsilon \in\{1,-1\}^{a}$ and set $W_{\epsilon}=W \cap U_{\epsilon}$. From [C], we have that

$$
\begin{aligned}
& W_{\epsilon}=\left\{\ell \in\left(W_{D} \oplus W_{M}\right) \cap U \mid \text { for each } \quad j=j_{r_{m}} \in M\right. \text {, } \\
& \left.\ell_{j}=\epsilon_{m}-p_{j}\left(\ell_{1}, \ell_{2}, \cdots, \ell_{j-1}\right)\right\}
\end{aligned}
$$

where $j \in M$ and $p_{j}$ is a rational nonsingular function on $U$.

Let $\epsilon \in\{1,-1\}^{a}$. Then from $[\mathbf{C}]$, there is a Zariski open subset $\Lambda_{\epsilon}$ of $W_{D}$ and a rational function $p_{\epsilon}: \Lambda_{\epsilon} \rightarrow W_{M}$ such that $W_{\epsilon}=\operatorname{graph}\left(p_{\epsilon}\right)$.

From $[\mathbf{C}]$, the projection of $U_{\epsilon}$ into $W_{D}$ parallel to $W_{J}$ defines a diffeomorphism $\pi_{\epsilon}$ of $W_{\epsilon}$ with $\Lambda_{\epsilon}$.

Remark 2.2. If $G$ is nilpotent, then $M$ is empty, $U_{\epsilon}=U, p_{\epsilon}=0$, and $\Lambda_{\epsilon}=W=U \cap W_{D}$ is a open subset in $W_{D}$. 
Let $O_{\lambda, \epsilon}$ be the coadjoint orbit via $\pi_{\epsilon}^{-1}(\lambda)$ for $\lambda \in \Lambda_{\epsilon}$ and let $\beta_{\lambda, \epsilon}$ be the canonical measure on $O_{\lambda, \epsilon}$. Identify $W_{D}$ with $\mathbb{R}^{n-2 k}$ via the basis $\left\{X_{i}^{*} \mid i \notin\right.$ $J\}$ and let $d \lambda$ be the Lebesgue measure on $W_{D}$. If $W_{D}=(0)$ the measure $d \lambda$ is a point mass measure. This is the case for the " $a x+b$ group" (see the example, paragraph 5).

Define $\Theta_{\epsilon}: V \times \Lambda_{\epsilon} \rightarrow U_{\epsilon}$ by $\Theta_{\epsilon}(v, \lambda)=P\left(v, \pi_{\epsilon}^{-1}(\lambda)\right)$. Then $\Theta_{\epsilon}$ is a diffeomorphism.

From 2.8 of $[\mathbf{C}]$, for any integrable function $F$ on $\mathfrak{g}^{*} / G$, we have

$$
\int_{\mathfrak{g}^{*} / G} F(O) d m_{\psi}(O)=\sum_{\epsilon} \int_{\Lambda_{\epsilon}} F\left(O_{\lambda, \epsilon}\right)\left|\psi\left(\pi_{\epsilon}^{-1}(\lambda)\right)\right|\left|\operatorname{Pf}\left(\pi_{\epsilon}^{-1}(\lambda)\right)\right|(2 \pi)^{-2 k} d \lambda
$$

where $\operatorname{Pf}\left(\pi_{\epsilon}^{-1}(\lambda)\right)$ denotes the Pffafian in $\pi_{\epsilon}^{-1}(\lambda)$.

Set $\left[\rho_{\lambda, \epsilon}\right]=K^{-1}\left(O_{\lambda, \epsilon}\right)$ for $\epsilon \in\{1,-1\}^{a}$ and $\lambda \in \Lambda_{\epsilon}$. For each nonzero rational function $\psi$ on $\mathfrak{g}^{*}$ satisfying $\psi(g . \ell)=\Delta(g)^{-1} \psi(\ell)$ for $g \in G$ and $\ell \in \mathfrak{g}^{*}$, let $A_{\psi, \lambda, \epsilon}$, denote the semi-invariant operator of weight $\Delta$ for the irreducible representation $\rho_{\lambda, \epsilon}$ corresponding to the restriction of $\psi$ to $O_{\lambda, \epsilon}$ (this operator is constructed in $[\mathbf{D R}]$ ).

In summary: Let $G$ be a connected, simply connected, and completely solvable Lie group. Let $\left\{X_{1}^{*}, X_{2}^{*}, \cdots, X_{n}^{*}\right\}$ be a Jordan-Hölder basis of $\mathfrak{g}^{*}$. Then, there is a finite collection of disjoint open subsets $U_{\epsilon}$ of $\mathfrak{g}^{*}$ and there is a subspace $W_{D}$ of $\mathfrak{g}^{*}$ such that for each $\epsilon, U_{\epsilon}$ is parametrized by a Zariski open subset $\Lambda_{\epsilon}$ of $W_{D}, \cup U_{\epsilon}$ is dense in $\mathfrak{g}^{*}$, and the complement of $\cup U_{\epsilon}$ has Plancherel measure zero. Let $\psi$ be a non empty rational function on $\mathfrak{g}^{*}$ such that $\psi(g . \ell)=\Delta(g)^{-1} \psi(\ell)$ for $g \in G$ and $\ell \in \mathfrak{g}^{*}$. For each $\epsilon$, there is a rational function $r_{\psi, \epsilon}$ on $W_{D}$ such that for any smooth compactly supported function $\phi$ on $G$, the function

$$
\lambda \rightarrow \operatorname{Tr}\left(A_{\psi, \lambda, \epsilon}^{-1 / 2} \rho_{\lambda, \epsilon}(\phi) A_{\psi, \lambda, \epsilon}^{-1 / 2}\right)\left|r_{\psi, \epsilon}(\lambda)\right|
$$

on $\Lambda_{\epsilon}$ is Lebesgue integrable. For any such $\phi$ we have

$$
\phi(e)=\sum_{\epsilon} \int_{\Lambda_{\epsilon}} \operatorname{Tr}\left(A_{\psi, \lambda, \epsilon}^{-1 / 2} \rho_{\lambda, \epsilon}(\phi) A_{\psi, \lambda, \epsilon}^{-1 / 2}\right)\left|r_{\psi, \epsilon}(\lambda)\right| d \lambda
$$

where $r_{\psi, \epsilon}(\lambda)=\psi\left(\pi_{\epsilon}^{-1}(\lambda)\right) \operatorname{Pf}\left(\pi_{\epsilon}^{-1}(\lambda)\right)(2 \pi)^{-2 k}$.

\section{Group Fourier Transform.}

We consider two cases:

First case: We suppose that $\mathfrak{g}^{\ell} \subset \mathfrak{g}_{n-1}$ for all $\ell \in W_{\epsilon}$ i.e. all the general position orbits are saturated with respect to $\mathfrak{g}_{n-1}$. We can choose a basis of $\mathfrak{g}$ in which the first $n-1$ vectors of the basis

$$
\left\{X_{1}(\ell), \ldots, X_{r}(\ell), \ldots, X_{m}(\ell), \ldots, X_{n-1}(\ell)\right\}
$$


for $\ell \in W_{\epsilon}$ depends on $\ell$, the $X_{i}(\ell)$ are in $\mathfrak{g}_{j}^{\ell_{j}}$ for certain $j$ with $\ell_{j}=\left.\ell\right|_{\mathfrak{g}_{j}}$, and $\mathfrak{g}_{j}^{\ell_{j}}=\left\{X \in \mathfrak{g}_{j} \mid a d^{*} X . \ell_{j}=0\right\}$. As $\mathfrak{g}^{\ell} \subset \mathfrak{g}_{n-1}$, the last vector of the basis does not depend on $\ell$. Let

$$
\mathfrak{B}_{W_{\epsilon}}(\ell)=\left\{X_{1}(\ell), \ldots, X_{r}(\ell), \ldots, X_{m}(\ell), \ldots, X_{n-1}(\ell), X_{n}\right\}
$$

be one such basis of $\mathfrak{g}$.

Remark that the index set $J_{1}$ for $G_{n-1}$ is equal to $J \backslash\left\{n, j_{1}\right\}$ and that $M_{1}=\left\{j_{r_{2}}, \cdots, j_{r_{a_{1}}}\right\}$ is a subset of $J_{1}$. For each $\epsilon_{1} \in\{1,-1\}^{a_{1}}$, the set $U_{\epsilon_{1}}$ is a nonempty open subset of $\mathfrak{g}_{n-1}^{*}$. Denote $W_{D_{1}}$ the subspace spanned by $\left\{X_{i}^{*} \mid i \notin J_{1}\right\}$ in $\mathfrak{g}_{n-1}^{*}$. Then, we have $W_{D_{1}}=W_{D} \oplus \mathbb{R} X_{j_{1}}^{*}$ and $W_{M_{1}}$ is the subspace spanned by $\left\{X_{j}^{*} \mid j \in M_{1}\right\}$.

Set $W_{\epsilon_{1}}=W_{1} \cap U_{\epsilon_{1}}$ where

$$
W_{1}=\left\{\ell_{1} \in\left(W_{D_{1}} \oplus W_{M_{1}}\right) \cap U_{1}|| q_{j}\left(\ell_{1}\right) \mid=1, j \in M_{1}\right\} .
$$

Now, by the corresponding theory for $G_{n-1}$ we have a Zariski open subset $\Lambda_{\epsilon_{1}}$ of $W_{D_{1}}$ and a rational function $p_{\epsilon_{1}}: \Lambda_{\epsilon_{1}} \rightarrow W_{M_{1}}$ such that $W_{\epsilon_{1}}=\operatorname{graph}\left(p_{\epsilon_{1}}\right)$.

Remark that $a_{1}=a-1$. In fact there is a case where $a_{1}=a$. If we start with any chain of ideals $0=\mathfrak{g}_{0} \subset \mathfrak{g}_{1} \subset \cdots \subset \mathfrak{g}_{i} \subset \cdots \subset \mathfrak{g}_{n-1} \subset \mathfrak{g}_{n}=\mathfrak{g}$, to avoid this case it suffices to choose a chain in such a manner that the chain passes through the nil-radical of $\mathfrak{g}$ when $\mathfrak{g}$ is non nilpotent. Also $\epsilon_{1}$ is obtained by deleting an element from $\epsilon$. Let $\Lambda_{\epsilon_{+}}^{\prime}$ denote the projection of $\Lambda_{\epsilon_{+}}$on $\mathfrak{g}_{n-1}^{*}$, and $\Lambda_{\epsilon_{-}}^{\prime}$ denote the projection of $\Lambda_{\epsilon_{-}}$on $\mathfrak{g}_{n-1}^{*}$.

The measure on $W_{\epsilon_{1}}$ is

$$
d \mu_{1}\left(\pi_{\epsilon_{1}}^{-1}\left(\lambda_{1}\right)\right)=\sum_{\epsilon_{1} \in\{1,-1\}^{a_{1}}}(2 \pi)^{-(2 k-2)} \psi_{1}\left(\pi_{\epsilon_{1}}^{-1}\left(\lambda_{1}\right)\right) \operatorname{Pf}\left(\pi_{\epsilon_{1}}^{-1}\left(\lambda_{1}\right)\right) d \lambda_{1}
$$

where $\operatorname{Pf}\left(\pi_{\epsilon_{1}}^{-1}\left(\lambda_{1}\right)\right)^{2}=\operatorname{det}\left(\pi_{\epsilon_{1}}^{-1}\left(\lambda_{1}\right)\left(\left[X_{i}, X_{j}\right]\right)_{i, j \in J_{1}}\right)$ with $\pi_{\epsilon_{1}}^{-1}\left(\lambda_{1}\right)=$ $\left.\pi_{\epsilon}^{-1}(\lambda)\right|_{\mathfrak{g}_{n-1}^{*}}$ and $\psi_{1}$ is a non empty rational function on $\mathfrak{g}_{n-1}^{*}$ such that we have $\psi_{1}\left(h . \ell_{1}\right)=\Delta(h)^{-1} \psi_{1}\left(\ell_{1}\right)$. Remark that, $\mathfrak{g}^{\ell_{n-1}}=\mathfrak{g}^{\ell} \oplus \mathbb{R} X_{j_{1}}$, $\left[X_{i}, X_{j}\right] \in \mathfrak{g}_{n-1}$ for $i, j$ in $J_{1}$, and $\ell\left(\left[X_{j_{1}}, \mathfrak{g}_{n-1}\right]\right)=0$.

Remark 3.1. For $\ell \in W_{\epsilon}$, let $A(\ell)=\left(\ell\left[X_{i}, X_{j}\right]\right)_{i, j \in J}$ be the skew-symmetric matrix.

$$
A(\ell)=\left(\begin{array}{ccc}
0 & \cdots 0 & \cdots \ell\left(\left[X_{n}, X_{j_{1}}\right]\right) \\
0 & & * \\
\vdots & A_{n-1}(\ell) & \vdots \\
\ell\left(\left[X_{j_{1}}, X_{n}\right]\right) & * & *
\end{array}\right)
$$

where $A_{n-1}(\ell)=\ell\left(\left[X_{i}, X_{j}\right]\right)_{i, j \in J_{1}}$.

Then: $\operatorname{det} A(\ell)^{\frac{1}{2}}=\left|\ell\left(\left[X_{j_{1}}, X_{n}\right]\right)\right|\left(\operatorname{det} A_{n-1}(\ell)^{\frac{1}{2}}\right)$.

That is, $\operatorname{Pf}(\ell)=\ell\left(\left[X_{j_{1}}, X_{n}\right]\right) \operatorname{Pf}\left(\ell_{n-1}\right)$ where $\ell_{n-1}=\left.\ell\right|_{\mathfrak{g}_{n-1}}$. 
Lemma 3.2. We suppose that $\mathfrak{g}^{\ell} \subset \mathfrak{g}_{n-1}$ for all $\ell \in W_{\epsilon}$. Let $\psi$ be a non empty rational function on $\mathfrak{g}^{*}$ such that $\psi(x . \ell)=\Delta(x)^{-1} \psi(\ell)$ for all $\ell \in W_{\epsilon}$ and $x \in G$. Then:

i. $\psi(\ell)=\psi\left(\ell^{\prime}\right)$ for $\ell^{\prime} \in \ell+\mathfrak{g}_{n-1}^{\perp}$.

ii. Let $\ell_{1} \in \mathfrak{g}_{n-1}^{*}$ and $\tilde{\ell}_{1}$ be an extension of $\ell_{1}$ to $\mathfrak{g}^{*}$. By taking $\psi_{1}\left(\ell_{1}\right)=$ $\psi\left(\tilde{\ell}_{1}\right)$ we obtain a rational function $\psi_{1}$ on $\mathfrak{g}_{n-1}^{*}$ verifying $\psi_{1}\left(h . \ell_{1}\right)=$ $\Delta_{G_{n-1}}(h)^{-1} \psi_{1}\left(\ell_{1}\right)$ for $h \in G_{n-1}$ and $\ell_{1} \in W_{\epsilon_{1}}$.

Proof. We have $G^{\ell} \subset G^{\ell_{n-1}}$ for $\ell \in W_{\epsilon}$ hence the stabilizer of $\ell_{n-1} \in \mathfrak{g}_{n-1}^{*}$ in $G$ is also equal to $G^{\ell_{n-1}}$.

Let $\ell^{\prime}=\ell+\gamma$ where $\gamma \in \mathfrak{g}_{n-1}^{\perp}$. Then $\ell^{\prime}=a . \ell$ with $a \in G^{\ell_{n-1}}$, hence we have that $\psi\left(\ell^{\prime}\right)=\psi($ a. $\ell)=\Delta(a)^{-1} \psi(\ell)$. We have to verify that $\Delta(a)=1$ if $a \in G^{\ell_{n-1}}$. But, $\Delta(a)=\Delta_{G_{n-1}}(a)$ since $G_{n-1}$ is normal in $G$. Moreover, $G_{n-1} / G_{n-1}^{\ell_{n-1}}$ has an invariant measure, so we have $\Delta_{G_{n-1}}(a)=\Delta_{G_{n-1}^{\ell_{n-1}}}(a)$. It suffices to see that $G_{n-1}^{\ell_{n-1}}$ is abelian since, the orbit of $\ell_{1}$ is of maximal dimension (see [B2], Chapter II). Hence $\psi\left(\ell^{\prime}\right)=\psi(\ell)$ which allows us to define $\psi_{1}$.

For all $h \in G_{n-1}$ and $\ell_{1} \in \mathfrak{g}_{n-1}^{*}$ we have

$$
\psi_{1}\left(h \cdot \ell_{1}\right)=\psi\left(\widetilde{h \cdot \ell_{1}}\right)=\psi\left(h \cdot \tilde{\ell}_{1}\right)=\Delta_{G}(h)^{-1} \psi\left(\tilde{\ell}_{1}\right)=\Delta_{G_{n-1}}(h)^{-1} \psi_{1}\left(\ell_{1}\right) .
$$

We express the measure $d \mu_{1}$ on $W_{\epsilon_{1}}$ in terms of local coordinates on $\mathfrak{g}_{n-1}^{*}$. From the above remark and the Lemma we have that

$$
\begin{gathered}
d \mu_{1}=\sum_{\epsilon_{1} \in\{1,-1\}^{a_{1}}}(2 \pi)^{2 k-2} \frac{1}{\psi_{1}\left(\pi_{\epsilon_{1}}^{-1}\left(\lambda_{1}\right)\right)} \frac{1}{\operatorname{Pf}\left(\pi_{\epsilon_{1}}^{-1}\left(\lambda_{1}\right)\right)} d \lambda_{1} \\
=\left(\sum_{\epsilon^{\prime}}(2 \pi)^{2 k-2} \frac{\pi_{\epsilon}^{-1}(\lambda)\left(\left[X_{j_{1}}, X_{n}\right]\right)}{\operatorname{Pf}\left(\pi_{\epsilon}^{-1}(\lambda)\right)} \frac{1}{\psi\left(\pi_{\epsilon}^{-1}(\lambda)\right)} d \lambda\right) d X_{j_{1}}^{*}
\end{gathered}
$$

where $\epsilon^{\prime}$ describes a part of $\{1,-1\}^{a}$.

This measure $W_{\epsilon_{1}} \subset \mathfrak{g}_{n-1}^{*}$ is a Plancherel measure on $\widehat{G_{n-1}}$, the unitary dual of $G_{n-1}$.

For $\ell \in W_{\epsilon}, \rho_{\ell}=\rho_{\lambda, \epsilon}=\operatorname{Ind}_{G_{n-1}}^{G} \rho_{\ell_{n-1}}$ is an induced representation of $G$, where $\ell_{n-1}=\ell_{\mathfrak{g}_{n-1}}$ and $\rho_{\ell_{n-1}}=\rho_{\lambda_{1}, \epsilon_{1}}$ is a representation of $G_{n-1}$. Let $\mathrm{C}^{\infty}(G, \rho)$ be the set of $f \in \mathrm{C}^{\infty}(G)$ with compact support modulo $G_{n-1}$ such that $f(h g)=\left(\rho_{\ell_{n-1}}(h)\right) f(g)$ for all $h \in G_{n-1}, g \in G$.

For all $\phi \in \mathrm{C}_{c}^{\infty}(G)$ and $\rho_{\ell} \in \widehat{G}$ such that $\ell \in W_{\epsilon}$, the group Fourier transform is defined by

$$
\widehat{\phi}_{\rho_{\ell}}=\int_{G} \phi(g) \rho_{\ell}(g) d g
$$


Set $\ell^{t}=A d^{*}(\exp (-t X)) \ell$. Remark that

$$
\rho_{\ell^{t}}(g)=\rho_{\ell}(\exp (t X) \cdot g \cdot \exp (-t X)) .
$$

Choose $X \in \mathfrak{g} \backslash \mathfrak{g}_{n-1}$. For all $s, t$ in $\mathbb{R}$, the action of $\phi \in \mathrm{C}_{c}^{\infty}(G)$ on $f \in \mathrm{H}_{\rho_{\ell}}$ gives us

$$
\left(\widehat{\phi}_{\rho_{\ell}} f\right)(\exp (t X))=\int_{G} \phi(g) \rho_{\ell}(g) f(\exp (t X)) d g .
$$

As the induced representation acts by right translation on $f \in \mathrm{H}_{\rho_{\ell}}$, we have

$$
\begin{aligned}
& \left(\widehat{\phi}_{\rho_{\ell}} f\right)(\exp (t X))=\int_{G} \phi(g) f(\exp (t X) \cdot g) d g \\
& =\int_{\mathbb{R}} \int_{G_{n-1}} \phi(h \cdot \exp (s X)) f(\exp (t X) \cdot h \cdot \exp (s X)) d h d s \\
& =\int_{\mathbb{R}} \int_{G_{n-1}} \phi(h \cdot \exp (s X)) f(\exp (t X) \cdot h \cdot \exp (-t X) \cdot \exp (t X) \cdot \exp (s X)) d h d s \\
& =\int_{\mathbb{R}} \int_{G_{n-1}} \phi(h \cdot \exp (s X)) f(\exp (t X) \cdot h \cdot \exp (-t X) \cdot \exp (t+s) X) d h d s \\
& =\int_{\mathbb{R}} \int_{G_{n-1}} \phi(h \cdot \exp (s X)) \rho_{\left(\ell^{t}\right)_{n-1}}(h) f(\exp (t+s) X) d h d s \\
& =\int_{\mathbb{R}} \int_{G_{n-1}} \phi^{s}(h) \rho_{\left(\ell^{t}\right)_{n-1}}(h) f(\exp (t+s) X) d h d s \\
& =\int_{\mathbb{R}}\left(\widehat{\phi}_{\rho_{\left(\ell^{t}\right)_{n-1}}}\right) f(\exp (t+s) X) d s
\end{aligned}
$$

where $\phi^{s}(h)=\phi(h \cdot \exp (s X))$.

For all $\alpha \in \mathbb{R}$ we set $f_{\alpha}(h \cdot \exp (s X))=e^{i \alpha s} f(h . \exp (s X))$. We have $f_{\alpha} \in$ $\mathrm{H}_{\rho_{\ell}}$, since $f$ is in $\mathrm{H}_{\rho_{\ell}}$.

Let ker $\rho_{\ell}$ denote the kernal of $\rho_{\ell}$ in $C^{*}(G)$, the $C^{*}$ - algebra of the group $G$. If $\phi \in \operatorname{ker} \rho_{\ell}$, then, from the above calculations, for all $f \in \mathrm{H}_{\rho_{\ell}}$ we have

$$
\begin{aligned}
0 & =\int_{\mathbb{R}} \widehat{\phi}_{\rho_{\left(\ell^{t}\right)_{n-1}}^{s}} f_{\alpha}(\exp (s+t) X) d s \\
& =\int_{\mathbb{R}} e^{i \alpha(s+t)} \widehat{\phi}_{\rho_{\left(\ell^{t}\right)_{n-1}}^{s}} f(\exp (s+t) X) d s \quad \forall \alpha \in \mathbb{R},
\end{aligned}
$$

which implies that $\widehat{\phi}_{\rho_{\left(\ell^{t}\right)_{n-1}}^{s}}=0$ for all $s \in \mathbb{R}$. Conversely, for all $s$ and $t$ in $\mathbb{R}$, if $\widehat{\phi}_{\rho_{\left(\ell^{t}\right)_{n-1}}^{s}}=0$ we have $\widehat{\phi}_{\rho_{\ell}}=0$ which implies that $\phi \in \operatorname{ker} \rho_{\ell}$. We have established an equivalence

$$
\phi \in \operatorname{ker} \rho_{\ell} \Longleftrightarrow\left(\widehat{\phi}_{\rho_{\left(\ell^{t}\right)_{n-1}}^{s}}=0 \forall s, t\right) .
$$


Second case: If all the general position orbits are not saturated with respect to $\mathfrak{g}_{n-1}$, we can choose a basis of $\mathfrak{g}$ in such a way that the last vector of the basis $X_{n}$ does not depend on $\ell$ and $X_{n}(\ell) \in \mathfrak{g}^{\ell}$. Let

$$
\mathfrak{B}_{W_{\epsilon}}(\ell)=\left\{X_{1}(\ell), \ldots, X_{r}(\ell), \ldots, X_{m}(\ell), \ldots, X_{n-1}(\ell), X_{n}(\ell)\right\}
$$

be one such basis of $\mathfrak{g}$ in which the $X_{i}(\ell)$ are in $\mathfrak{g}_{j}^{\ell_{j}}$ for certain $j$ with $\ell_{j}=\left.\ell\right|_{\mathfrak{g}_{j}}$.

Lemma 3.3. Assume that $\mathfrak{g}^{\ell} \not \subset \mathfrak{g}_{n-1}$ for all $\ell \in W_{\epsilon}$. Let $\psi$ be a non empty rational function on $\mathfrak{g}^{*}$ such that $\psi(x . \ell)=\Delta(x)^{-1} \psi(\ell)$ for all $\ell \in$ $W_{\epsilon}$ and $x \in G$. Let $\ell_{1} \in \mathfrak{g}_{n-1}^{*}$ and $\tilde{\ell}_{1}$ be an extension of $\ell_{1}$ to $\mathfrak{g}^{*}$. By letting $\psi_{1}\left(\ell_{1}\right)=\psi\left(\tilde{\ell}_{1}\right)$ we obtain a rational function $\psi_{1}$ on $\mathfrak{g}_{n-1}^{*}$ satisfying $\psi_{1}\left(h . \ell_{1}\right)=\Delta(h)^{-1} \psi_{1}\left(\ell_{1}\right)$ for all $h \in G_{n-1}$.

Proof. For all $\ell \in \mathfrak{g}^{*}$ and $\alpha \in \mathbb{R}$ we have $\ell_{\alpha}=\ell+\alpha X_{n}^{*}$ and $\mathfrak{g}^{*}=\mathfrak{g}_{n-1}^{*} \oplus \mathbb{R} X_{n}^{*}$. For all $h \in G_{n-1}$, we have $h . \ell_{\alpha}=h . \ell+\alpha X_{n}^{*}$ since $G . X_{n}^{*}=X_{n}^{*}$. By choosing $\alpha=0$, we have $\ell_{0}=\ell+0 X_{n}^{*}$ and $h . \ell_{0}=h . \ell$. Hence, $\psi_{1}\left(\ell_{1}\right)=\psi\left(\tilde{\ell}_{1}\right)$ and

$$
\psi_{1}\left(h \cdot \ell_{1}\right)=\psi\left(h \cdot \ell_{1}\right)=\Delta(h)^{-1} \psi\left(\ell_{1}\right)=\Delta_{G_{n-1}}(h)^{-1} \psi_{1}\left(\ell_{1}\right) .
$$

Remark that the set of indices $J_{1}$ for $G_{n-1}$ is equal to $J$. In this case as $\mathfrak{g}^{\ell}=\mathfrak{g}^{\ell_{n-1}}+\mathbb{R} X_{n}$ we have $W_{D}=W_{D_{1}}+\mathbb{R} X_{n}$, where $W_{D_{1}}$ is the subspace of $\mathfrak{g}_{n-1}^{*}$ corresponding to $W_{D}$ in $\mathfrak{g}^{*}$. Moreover, $\Lambda_{\epsilon}=\Lambda_{\epsilon_{1}}+\mathbb{R} X^{*}$. The Plancherel measure over $\widehat{G}$ can be written as;

$$
\begin{aligned}
d \mu(\ell) & =\sum_{\epsilon}(2 \pi)^{2 k} \frac{1}{\psi\left(\pi_{\epsilon}^{-1}(\lambda)\right)} \frac{1}{\operatorname{Pf}\left(\pi_{\epsilon}^{-1}(\lambda)\right)} d X_{1}^{*} \cdots d X_{n-2 k-1}^{*} d X_{n}^{*} \\
& =\left(\sum_{\epsilon_{1}}(2 \pi)^{2 k} \frac{1}{\psi_{1}\left(\pi_{\epsilon_{1}}^{-1}\left(\lambda_{1}\right)\right)} \frac{1}{\operatorname{Pf}\left(\pi_{\epsilon_{1}}^{-1}\left(\lambda_{1}\right)\right)} d X_{1}^{*} \cdots d X_{n-2 k-1}^{*}\right) d X_{n}^{*} \\
& =d \mu_{1} \times d X_{n}^{*} .
\end{aligned}
$$

For $\ell=\pi_{\epsilon}^{-1}(\lambda) \in W_{\epsilon}$, and $\alpha \in \mathbb{R}$ we let $\ell_{\alpha}=\ell+\alpha X^{*}$. Hence, $\ell_{\alpha}(X)=$ $\ell(X)+\alpha$ and $\rho_{\ell_{\alpha}}=\rho_{\ell} \otimes \chi_{\alpha}$ with $\chi_{\alpha}(h . \exp (s X))=e^{i \alpha s}$ for all $h \in G_{n-1}$. 
The restriction of $\rho_{\ell_{\alpha}}$ to $G_{n-1}$ is irreducible and equivalent to $\rho_{\ell_{n-1}}$ for all $\alpha \in \mathbb{R}$. For all $\xi, \eta \in \mathrm{H}_{\rho_{\ell}}=\mathrm{H}_{\rho_{\ell_{\alpha}}}$ we have

$$
\begin{aligned}
& \left\langle\widehat{\phi}_{\rho_{\ell_{\alpha}}} \xi, \eta\right\rangle=\int_{G}\left\langle\rho_{\ell_{\alpha}}(g) \xi, \eta\right\rangle \phi(g) d g \\
& =\int_{G}\left\langle\rho_{\ell} \otimes \chi_{\alpha}(g) \xi, \eta\right\rangle \phi(g) d g \\
& =\int_{\mathbb{R}} \int_{G_{n-1}}\left\langle\rho_{\ell} \otimes \chi_{\alpha}(\exp (s X) \cdot h) \xi, \eta\right\rangle \phi(\exp (s X) \cdot h) d h d s \\
& =\int_{\mathbb{R}} \int_{G_{n-1}}\left\langle e^{i \alpha s} \rho_{\ell}(\exp (s X)) \rho_{\ell_{n-1}}(h) \xi, \eta\right\rangle \phi(\exp (s X) \cdot h) d h d s \\
& =\int_{\mathbb{R}} e^{i \alpha s}\left\langle\rho_{\ell}(\exp (s X)) \widehat{\phi}_{\rho_{\ell_{n-1}}^{s}} \xi, \eta\right\rangle d s
\end{aligned}
$$

where $\phi^{s}(h)=\phi(\exp (s X) \cdot h)$. Hence we have expressed $\widehat{\phi}_{\rho_{\ell_{\alpha}}}$ with the help of $\widehat{\phi}_{\rho_{\ell_{n-1}}}$.

\section{Weak Paley-Wiener Property.}

Theorem 4.1. Let $G$ be a connected, simply connected, and completely solvable Lie group with the unitairy dual $\widehat{G}$, and let $\phi$ be a bounded, measurable and compactly supported function (i.e. $\phi \in L_{c}^{\infty}(G)$ ). Assume that there is a subset $E \subset \widehat{G}$ with positive Plancherel measure such that $\widehat{\phi}_{\rho}=0$ for all $\rho \in E$, where $\widehat{\phi}_{\rho}$ is the group Fourier transform of $\phi$. Then $\phi=0$ almost every where on $G$.

Proof. We proceed by induction on the dimension $n$ of $G$. The result is true if the dimension of $G$ is one, since $G \cong \mathbb{R}$. Assume that the result is true for all groups of dimension $n-1$. We can assume that $E$ is contained in $W_{\epsilon}$ (it suffices to take $E$ as the finite union of $E \cap W_{\epsilon}$ ).

First case: $\mathfrak{g}^{\ell} \subset \mathfrak{g}_{n-1}$ for all $\ell \in W_{\epsilon}$. Let $\phi \in \mathrm{C}_{c}^{\infty}(G)$. By hypothesis, for all $\rho_{\ell}$, such that $\ell \in E$ we have $0=\widehat{\phi}_{\rho_{\ell}}$; we will show that $\phi=0$ almost every where on $G$.

Notice that for all $\epsilon_{1} \in\{-1,1\}^{a_{1}}$, the associated set $\Lambda_{\epsilon_{1}}$ corresponds to two sets $\Lambda_{\epsilon_{+}}$and $\Lambda_{\epsilon_{-}}, \epsilon_{ \pm} \in\{-1,1\}^{a}$ in $W_{D}$. If $\Lambda_{\epsilon_{+}}^{\prime}$ and $\Lambda_{\epsilon_{-}}^{\prime}$ are the projections of $\Lambda_{\epsilon_{+}}$and $\Lambda_{\epsilon_{-}}$on $\mathfrak{g}_{n-1}^{*}$ such that $\Lambda_{\epsilon_{1}}^{\prime}=(\exp \mathbb{R} X) \cdot \Lambda_{\epsilon_{+}}^{\prime} \cup(\exp \mathbb{R} X) . \Lambda_{\epsilon_{-}}^{\prime}$ and $T_{\ell}=\left\{\exp t X . \ell_{n-1} \mid t \in \mathbb{R}\right\}$ are contained in the projection of $\Lambda_{\epsilon_{+}}$or in $\Lambda_{\epsilon_{-}}, \Lambda_{\epsilon_{1}}^{\prime}$ is a Zariski open set in $\Lambda_{\epsilon_{1}}$.

From paragraph 3 we have that

$$
\phi \in \operatorname{ker} \rho_{\ell} \Longleftrightarrow\left(\widehat{\phi}_{\rho_{\left(\ell^{t}\right)_{n-1}}^{s}}=0 \forall s, t\right) .
$$


By hypothesis, $\widehat{\phi_{\rho_{\ell}}}=0$ for all $\ell \in E$ and from the above equivalence we have

$$
\widehat{\phi}_{\rho_{\left(\ell^{t}\right)_{n-1}}^{s}}=0
$$

for all $s, t$ in $\mathbb{R}$. This relation tells us that a set $A$ contained in $\Lambda_{\epsilon_{+}} \cup \Lambda_{\epsilon_{-}}$ has positive Plancherel measure if and only if the set $\cup_{\rho_{\ell} \in A} T_{\ell}$ has positive Plancherel measure in $\Lambda_{\epsilon_{1}}$.

In applying this remark to the set $E$, we obtain

$$
\widehat{\phi}_{\rho_{\left(\ell^{t}\right)_{n-1}}^{s}}^{s}=0
$$

for all $\rho_{\ell_{n-1}}$ in $E^{\prime} \subset \widehat{G}_{n-1}$ with positive Plancherel measure.

By the induction hypothesis $\phi^{s}=0$ almost everywhere on $G_{n-1}$, which implies that $\phi=0$ almost everywhere on $G$ by using Fubini's theorem.

Second case: $\mathfrak{g}^{\ell} \not \subset \mathfrak{g}_{n-1}$ for all $\ell \in W_{\epsilon}$. Let $\phi \in \mathrm{C}_{c}^{\infty}(G)$. By hypothesis, for all $\rho_{\ell}$, such that $\ell \in E$ we have $\widehat{\phi}_{\rho_{\ell}}=0$; let us show that $\widehat{\phi}_{\rho_{\ell}}=0$ for all $\ell \in W_{\epsilon}$.

Let $\ell \in E$. For all $\alpha \in \mathbb{R}$ we have

$$
\left\langle\widehat{\phi}_{\rho_{\ell_{\alpha}}} \xi, \eta\right\rangle=\int_{\mathbb{R}} e^{i \alpha s}\left\langle\rho_{\ell}(\exp (s X)) \widehat{\phi}_{\rho_{\ell_{n-1}}^{s}} \xi, \eta\right\rangle d s
$$

hence

$$
\widehat{\phi}_{\rho_{\ell_{\alpha}}}=\int_{\mathbb{R}} e^{i \alpha s} \rho_{\ell}(\exp (s X)) \widehat{\phi}_{\rho_{\ell_{n-1}}}^{s} d s
$$

Set

Hence

$$
\Psi(s)=\rho_{\ell}(\exp (s X)) \widehat{\phi}_{\rho_{\ell_{n-1}}}^{s} .
$$

$$
\begin{aligned}
\widehat{\phi}_{\rho_{\ell_{\alpha}}} & =\int_{\mathbb{R}} \Psi(s) e^{i \alpha s} d s \\
& =\widehat{\Psi}(\alpha) .
\end{aligned}
$$

By hypothesis, for all $\ell \in E$ we have $\widehat{\phi}_{\rho_{\ell}}=0$. The above calculation tells us that there exists a set $E^{\prime} \subset E$ with positive Plancherel measure such that $\widehat{\Psi}(\alpha)=0$ for $\alpha$ belonging to a set of reals with positive Lebesgue measure and $\ell \in E^{\prime}$. Hence $\Psi=0$ almost everywhere; consequently we have $\Psi(s)=0$ for almost every $s \in \mathbb{R}$. Hence

$$
0=\widehat{\phi}_{\rho_{\ell_{\alpha}}}=\int_{\mathbb{R}} e^{i \alpha s} \rho_{\ell}(\exp (s X)) \widehat{\phi}_{\rho_{\ell_{n-1}}}^{s} d s
$$

for all $\alpha$ in $\mathbb{R}$, which implies that $\widehat{\phi}_{\rho_{\ell_{n-1}}}=0$ for all $\ell_{n-1}$ in $E_{1}$ (path of $E$ on $\mathfrak{g}_{n-1}^{*}$ ) with positive Plancherel measure on $\widehat{G_{n-1}}$. By using the induction hypothesis $\widehat{\phi}_{\rho_{\ell_{n-1}}}=0$ for almost all $\ell_{n-1} \in W^{\prime}$ (path of $W_{\epsilon}$ on $\mathfrak{g}_{n-1}^{*}$ ). Hence, $0=\widehat{\phi}_{\rho_{\ell}}$ for almost all $\ell \in W_{\epsilon}$ (from the above calculation of $\widehat{\phi}_{\rho_{\ell_{\alpha}}}$ ). 
Hence $\widehat{\phi}_{\rho}=0$ for almost all $\rho$ relating with the Plancherel measure. By the Plancherel formula for completely solvable Lie groups, we have

$$
\phi(e)=\sum_{\epsilon} \int_{\Lambda_{\epsilon}} \operatorname{Tr}\left(A_{\psi, \lambda, \epsilon}^{-1 / 2} \rho_{\lambda, \epsilon}(\phi) A_{\psi, \lambda, \epsilon}^{-1 / 2}\right)\left|r_{\psi, \epsilon}(\lambda)\right| d \lambda
$$

which implies that $\phi=0$.

Now, we consider $\phi \in L_{c}^{\infty}(G)$. Let $\left\{f_{n}\right\}_{n}$ be an apporoximate identity in $C_{c}^{\infty}(G)$. For all integers $n, f_{n} * \phi \in C_{c}^{\infty}(G)$. Let $\rho \in E$. If $\widehat{\phi}_{\rho}$ vanishes, then $\left(\widehat{f_{n} * \phi}\right)_{\rho}$ also vanishes. Hence by what precedes, $f_{n} * \phi=0$ (for all integers $n)$. But, $\left(f_{n} * \phi\right)_{n \in N}$ converges to $\phi$ in $L^{1}(G)$, which implies that $\phi=0$ almost everywhere on $G$.

\section{Example: The $a x+b$ Group.}

Consider the group

$$
G=\left\{\left(\begin{array}{ll}
a & b \\
0 & 1
\end{array}\right) \mid a>0, b \in \mathbb{R}\right\} .
$$

We use the notation

$$
(a, b)=\left(\begin{array}{ll}
a & b \\
0 & 1
\end{array}\right)
$$

The Matrix multiplication gives:

$$
\left(a_{1}, b_{1}\right)\left(a_{2}, b_{2}\right)=\left(a_{1} a_{2}, a_{1} b_{2}+b_{1}\right)
$$

and the inverse

$$
(a, b)^{-1}=\left(a^{-1},-b a^{-1}\right) .
$$

Let $H=(1, b)$ be the derived group of $G$ which is identified with $\mathbb{R}$. Let $y \in \mathbb{R}, \chi_{y}$ the character of $H$ defined by $\chi_{y}((1, b))=e^{i b y}$.

Remark that $(a, b)=(1, b)(a, 0)$. Let $\rho_{y}=\operatorname{Ind}_{H}^{G} \chi_{y}$ be the induced representation of $G$. This representation is realized in the space $L^{2}(\mathbb{R})$. Recall that for all $y>0, \rho_{y}$ is equivalent to $\rho_{1}$ and we denote by $\rho_{+}$the class of the representation $\rho_{1}$. If $y<0, \rho_{y}$ is equivalent to $\rho_{-1}$; we denote by $\rho_{-}$the equivalence class of this representation.

The Lie algebra $\mathfrak{g}$ of $G$ is the set of matrices

$$
\mathfrak{g}=\left\{\left(\begin{array}{ll}
x & y \\
0 & 0
\end{array}\right),(x, y) \in \mathbb{R}^{2}\right\} .
$$

In the basis

$$
X=\left(\begin{array}{ll}
1 & 0 \\
0 & 0
\end{array}\right) \text { and } Y=\left(\begin{array}{ll}
0 & 1 \\
0 & 0
\end{array}\right)
$$

we have $[X, Y]=Y$. With the basis $X$ and $Y$ we have

$$
A d(a, b)=\left(\begin{array}{cc}
1 & 0 \\
-b & a
\end{array}\right)
$$


Also in the dual basis $\left\{X^{*}, Y^{*}\right\}$

$$
A d^{*}(a, b)=\left(\begin{array}{cc}
1 & b a^{-1} \\
0 & a^{-1}
\end{array}\right) .
$$

For $\ell=\alpha X^{*}+\beta Y^{*} \in \mathfrak{g}^{*}$ the orbits of $G$ in $\mathfrak{g}^{*}$ are the upper half plane $\beta>0$, the lower half plane $\beta<0$ and the points $(\alpha, 0)$.

Let $\mathfrak{B}=\{X, Y\}$ be the basis of $\mathfrak{g}$ defined above, and $\mathfrak{B}^{*}=\left\{X^{*}, Y^{*}\right\}$ the dual basis of $\mathfrak{g}^{*}$. There exists a set $J=\left\{j_{1}, j_{2}\right\} \subseteq\{1,2\}$ and $M=\left\{j_{2}\right\}$ a subset of $J$, so that $\left.V \subset \mathbb{R}^{2}, V=\right] 0, \infty\left[\times \mathbb{R}\right.$. We have $W_{D}=(0)$ and $W_{M}$ is spanned by the vector $\left\{X_{j_{2}}^{*} \mid j_{2} \in M\right\}$.

The Zariski open sets $U_{+}$and $U_{-}$are the half planes of $\mathfrak{g}^{*}$ defined above and $U=U_{+} \cup U_{-}$. Here, $a=1$ and $\epsilon \in\{1,-1\}$.

Since there are only two orbits, the set

$$
W=\left\{\ell \in W_{M} \cap U|| q_{j_{2}}(\ell) \mid=1, j_{2} \in M\right\}
$$

is a union of two points in $\mathfrak{g}^{*}$. We have $W_{+}=W \cap U_{+}$and $W_{-}=W \cap U_{-}$. Let $\epsilon \in\{1,-1\}$. In this case the Zariski open set is $\Lambda_{\epsilon}=\Lambda_{+}$or $\Lambda_{\epsilon}=\Lambda_{-}$of $W_{D}$, which reduces to a point.

In this particular case we can prove weak Paley-Wiener property by direct calculations.

Let $\phi \in \mathrm{C}_{c}^{\infty}(G), f \in L^{2}\left(\mathbb{R}_{+}^{*}\right)$ and $(t, 0) \in \mathbb{R}_{+}^{*}$ : then

$$
\begin{aligned}
\left(\widehat{\phi}_{\rho_{\ell}} f\right)(t) & =\int_{G} \phi((a, b)) \rho_{\ell}((a, b)) f(t) a^{-2} d a d b \\
& =\int_{G} \phi((a, b)) f\left((a, b)^{-1}(t, 0)\right) a^{-2} d a d b \\
& =\int_{\mathbb{R}_{+}^{*}} \int_{\mathbb{R}} \phi((a, b)) f\left(\left(a^{-1} t,-b a^{-1}\right)\right) a^{-2} d a d b \\
& =\int_{\mathbb{R}_{+}^{*}} \int_{\mathbb{R}} \phi((a, b)) f\left(\left(a^{-1} t, 0\right)\left(1,-b t^{-1}\right)\right) a^{-2} d a d b \\
& =\int_{\mathbb{R}_{+}^{*}}\left(\int_{\mathbb{R}} \phi((a, b)) \chi_{y}\left(\left(1,-b t^{-1}\right)\right) d b\right) f\left(\left(a^{-1} t, 0\right)\right) a^{-2} d a \\
& =\int_{\mathbb{R}_{+}^{*}}\left(\int_{\mathbb{R}} \phi^{a}(b) e^{-i b y t^{-1}} d b\right) f\left(\left(a^{-1} t, 0\right)\right) a^{-2} d a \\
& =\int_{\mathbb{R}_{+}^{*}} \widehat{\phi}_{\chi_{y t}-1}^{a} f\left(\left(a^{-1} t, 0\right)\right) a^{-2} d a,
\end{aligned}
$$

where $\phi^{a}(b)=\phi((a, b))$.

Remark that $\phi^{a} \in \mathrm{C}_{c}^{\infty}(\mathbb{R})$. By hypothesis we have $\widehat{\phi}_{\rho_{\ell}}=0$ for all $\ell \in E$. The above calculation implies that for all $a>0$ we have $\widehat{\phi}_{\chi_{y t}-1}^{a}=0$ for almost all $t>0$ and for fixed $y$. 
As $\phi^{a} \in \mathrm{C}_{c}^{\infty}(\mathbb{R}), \widehat{\phi}_{\chi_{y t^{-1}}}^{a}$ extends as an entire function over $\mathbb{C}$. $\widehat{\phi}_{\chi_{y t^{-1}}}^{a}$ vanishes on a set in which the Plancherel measure $d \mu_{1}$ is positive hence by the classical Paley-Wiener theorem, we can conclude that $\phi^{a}=0$, and then $\phi=0$ almost everywhere on $G$.

Acknowledgement. This article is part of the author's Ph.D thesis. The author is indebted to her advisor Professor Gérard Grélaud, for his help with this work.

\section{References}

[B1] P. Bernat and al, Sur les représentations unitaires des groupes de Lie résolubles, Ann. Sci. EC. Norm. Sup, 3ème série, 82 (1965).

[B2] , Représentations des groupes de Lie résolubles, Paris, Dunod, 1972.

[C] B.N. Currey, An explicit Plancherel formula for completely solvable Lie groups, Michigan Math Journal, 38 (1991), 75-87.

[DR] M. Duflo and M. Raïs, Sur l'analyse harmonique sur les groupes de Lie résolubles, Ann. Sci. École. Norm. Sup., 9(4) (1976), 107-144.

[GG1] G. Garimella, Un théorème de Paley-Wiener pour les groupes de Lie nilpotents, Journal of Lie Theory, 5 (1995), 165-172.

[GG] - Théorèmes de Paley-Wiener. Opérateurs différentiels invariants sur les groupes de Lie nilpotents, Ph.D thesis, Université de Poitiers, 1997.

$[\mathrm{Pu}]$ L. Pukanszky, On the characters and the Plancherel formula of nilpotent Lie groups, J. Func. Anal., 1 (1967), 255-280.

Received July 21, 1997 and revised October 22, 1997.

Indian Statistical Institute

BANGALORE - 560059

INDIA

E-mail address: gaya@ns.isibang.ac.in 